\title{
Size and Power Properties of Some Test Statistics for Testing the Population Correlation Coefficient $\rho$
}

\section{Shipra Banik ${ }^{1}$ and Golam Kibria BM ${ }^{2 \star}$}

${ }^{1}$ Department of Physical Sciences Independent University, Bangladesh Dhaka 1229, Bangladesh ${ }^{2}$ Department of Mathematics and Statistics Florida, International University Miami, FL 33199, USA

\begin{abstract}
Correlation measures the strength of association between two variables, which plays an important role in various fields, such as Health Science, Economics, Finance, Engineering, Environmental science among others. Several tests for testing the population correlation coefficient are proposed in a literature by various researchers at different time points. This paper evaluates the performance of some of the prominent test statistics for testing the population correlation coefficient based on empirical size and power of the tests. Some bivariate distributions, such as normal, lognormal, gamma and chi-square are considered to compare the performance of the test statistics. We believe that the findings of this paper will make an important contribution to select some good test statistics to find the relationship between two variables.
\end{abstract}

Keywords: Bivariate distribution; Bootstrapping; Correlation coefficients; Hypothesis tests; Monte Carlo simulation; Power; Size

\section{Introduction}

One of the most useful statistical tools for quantifying the relationship between two continuous variables is the coefficient of correlation that developed by Pearson [1] from a related idea introduced by Galton [2]. In statistics, Pearson's correlation coefficient is used to find the linear relationship between two quantitative variables (say) $\mathrm{X}$ and $\mathrm{Y}$. It gives a value between -1 and +1 inclusive, where -1 indicates a perfect negative correlation, 0 is no correlation and +1 indicates a perfect positive correlation between X and Y. Since the population correlation coefficient, $\rho$ is usually unknown, it is necessary to estimate it by estimator, $r$ from the observed data or sample information. Even the sample correlation coefficient $(r)$ is a biased estimator of population correlation coefficient $\rho$, the biasness disappears with the increase of sample size. When there is a question of estimation, its estimation accuracy and thus the validity through the hypothesis testing is essential. Several researchers considered several confidence intervals for estimating the population correlation coefficient $\rho$ [3]. However, a comparison of several test statistics for testing the population correlation coefficient is limited in literature. In this paper, we have made an attempt to consider several test statistics for testing the population correlation coefficient. Since, a theoretical comparison among the test procedures is not possible, a simulation study will be conducted to compare the performance of the test statistics based on empirical size and power of the test. We believe that the findings of this study will make an important contribution to literature to choose appropriate test statistics for testing the population correlation coefficient for practitioners.

The paper is organized as follows: One proposed and some existing methods for testing the population correlation coefficient are described in section 2. A Monte Carlo simulation study along with results is discussed in section 3. Finally, some concluding remarks are given in section 4 .

\section{Methods for Testing the Population Correlation Coefficient}

Suppose we are interested to find the linear relationship between two variables $\mathrm{X}$ and $\mathrm{Y}$. Then the population correlation coefficient between two variables $\mathrm{X}$ and $\mathrm{Y}$ is denoted by $\rho$ and is defined by

$$
\tilde{\mathrm{n}}=\frac{\operatorname{Cov}(\mathrm{X}, \mathrm{Y})}{\sqrt{\operatorname{Var}(\mathrm{X}) \operatorname{Var}(\mathrm{Y})}}=\frac{{ }^{\sigma} \mathrm{XY}}{\sqrt{{ }^{2} \mathrm{X}^{\sigma^{2} \mathrm{Y}}}} .
$$

The corresponding sample correlation coefficient is defined by

$$
r=\frac{\hat{\sigma} x y}{\sqrt{\hat{\sigma}^{2} x \hat{\sigma}^{2} y}} \text {. }
$$

It can be shown that $-1 \leq \rho \leq 1$. A value of 1 implies that a linear equation describes the relationship between $\mathrm{X}$ and $\mathrm{Y}$ perfectly, with all data points lying on a line for which $\mathrm{Y}$ increases as $\mathrm{X}$ increases. A value of -1 implies that all data points lie on a line for which $\mathrm{Y}$ decreases as $\mathrm{X}$ increases. A value of 0 implies that there is no linear association between $\mathrm{X}$ and $\mathrm{Y}$. Several methods for testing for population correlation coefficient, $H_{0}: \rho=0$ vs. $H_{1}: \rho \neq 0$ are given as follows.

\section{The classical test statistic}

Suppose, the sample correlation coefficient, $r$ is a point estimator of $\rho$. The distribution of $r$ when $\rho$ is zero was for the first time studied by a student [4]. Thus, a common test is that of whether or not a linear relationship exists between two variables $\mathrm{X}$ and $\mathrm{Y}$. The test statistic is defined as follows:

$$
t_{r}=\left(\sqrt{(n-2)} \sqrt{\frac{r^{2}}{1-r^{2}}}\right.
$$

where $n$ is the sample size and $(n-2)$ is the degrees of freedom(df). Thus the critical value for this test statistic can be obtained from $t$-distribution with $(n-2)$ degrees of freedom.

*Corresponding author: Golam Kibria BM, Department of Mathematics and Statistics Florida, International University Miami, FL 33199, USA, Tel: +1 305-3482000; E-mail: kibriag@fiu.edu

Received May 31, 2017; Accepted June 16, 2017; Published June 30, 2017

Citation: Banik S, Golam Kibria BM (2017) Size and Power Properties of Some Test Statistics for Testing the Population Correlation Coefficient $\rho$. J Biom Biostat 8: 353. doi: 10.4172/2155-6180.1000353

Copyright: (c) 2017 Banik S, et al. This is an open-access article distributed under the terms of the Creative Commons Attribution License, which permits unrestricted use, distribution, and reproduction in any medium, provided the original author and source are credited. 
Citation: Banik S, Golam Kibria BM (2017) Size and Power Properties of Some Test Statistics for Testing the Population Correlation Coefficient $\rho$. J Biom Biostat 8: 353. doi: 10.4172/2155-6180.1000353

Page 2 of 10

\section{Fisher's large sample test statistic}

Since the sampling distribution of Pearson's $r$ is not normally distributed, Pearson's $r$ is converted to Fisher's $z$ and the test statistic for testing $\mathrm{H}_{0}: \rho=0$ vs. $\mathrm{H}_{1}: \rho \neq 0$ is computed using Fisher's [5] transformation and is given as follows:

$$
t_{z}=\sqrt{n-3}\left(\mathrm{z}-z_{0}\right)
$$

Where $\mathrm{z}=\frac{1}{2} \log \left(\frac{1+r}{1-r}\right)$ and $z_{0}$ is the value of $\mathrm{z}$ under the null hypothesis where $z_{0}=\frac{1}{2} \log \left(\frac{1+\rho_{0}}{1-\rho_{0}}\right)$

The distribution of $t_{z}$ has a standard normal distribution.

\section{Gorsuch and Lehmann test statistics}

To improve the performances of the classical statistic, Gorsuch and Lehmann [6] modified the classical statistic and the Fisher statistic and proposed the following four statistics for testing $\mathrm{H}_{0}: \rho=0 v s . \mathrm{H}_{1}: \rho \neq 0$ based on different standard errors of $r$ :

Modified classical statistics:

$$
\begin{aligned}
& t_{G L 1}=\left(\sqrt{(\mathrm{n}-1)}\left(\frac{r}{1-r^{2}}\right)\right. \\
& t_{G L 2}=\left(\sqrt{(\mathrm{n}-1)}\left(\frac{r}{1-r^{2}}\right)\right.
\end{aligned}
$$

Where $\mathrm{r}=\frac{e^{2 \hat{\Psi}}-1}{e^{2 \hat{\Psi}}+1}, \hat{\Psi}=\frac{1}{2} \log \left(\frac{1+r}{1-r}\right)$ the critical value of $t_{G L 1}$ is assumed to be 2 (details see Gorsuch and Lehmann [6]) and the distribution of $t_{G L 2}$ follows t distribution with $(n-1) \mathrm{df}$.

Modified Fisher statistics:

$$
\begin{aligned}
& t_{G L 3}=\sqrt{n-3}\left(\mathrm{z}-\mathrm{z}_{0}\right) \\
& t_{G L 4}=\sqrt{n-3}\left(\mathrm{z}-\mathrm{z}_{0}\right)
\end{aligned}
$$

where the critical value of $t_{G L 3}$ is assumed to be 2 (details see Gorsuch and Lehmann [6]) and $t_{G L 4}$ has a t-distribution with (n-1) degrees of freedom.

\section{Proposed test statistic}

We know that $r=b_{1} \times \frac{s_{x}}{s_{y}}$, where b1 is estimator of $\beta_{1}$ for the model $y_{t}=\beta_{0}+\beta_{1} x_{t}+e_{t}, \beta_{0}$ is the constant, $\beta_{1}$ is the regression coefficient of $y$ on $x$ and $e_{t} \sim N\left(0, \sigma^{2}\right)$. The test statistic for testing $H_{0}: \rho=0 v s . H_{1}: \rho \neq 0$ is given by

$$
t_{S K}=\frac{r}{\sqrt{\frac{M S E}{\sum_{i=1}^{n}\left(\mathrm{y}_{i-} \overline{\mathrm{y}}\right)^{2}}}}
$$

The distribution of $t_{S K}$ has t-distribution with (n-2) df.

\section{Parametric bootstrap test statistic}

Let $\mathrm{x}^{(*)}=x_{1}^{(*)}, x_{2}^{(*)}, . ., x_{n}^{(*)}$ and $y^{(*)}=y_{1}^{(*)}, \mathrm{y}_{2}^{(*)}, . ., \mathrm{y}_{n}^{(*)}$ where $\mathrm{i}^{\text {th }}$ random samples are denoted by $\mathrm{x}^{(\mathrm{i})}$ and $\mathrm{y}^{(\mathrm{i})}$ for $\mathrm{i}=1,2, \ldots, \mathrm{B}$ and $\mathrm{B}$ is the number of bootstrap samples [7]. The test statistic for testing $\mathrm{H}_{0}: \rho=0$ vs. $\mathrm{H}_{1}: \rho$ $\neq 0$ is given by

$$
t_{p b o o t}=\left(\sqrt{(\mathrm{n}-2)} \sqrt{\frac{r^{2}}{1-r^{2}}}\right.
$$

Where critical values of the above statistic is the $t_{(\mathrm{n}-2)}^{*}, \frac{\alpha}{2}$ which is the $(\alpha / 2)^{\text {th }}$ sample quintiles of $t_{p b o o t}$

\section{Parametric bootstrap Fisher $\mathrm{z}$ test statistic}

The test statistic for testing $\mathrm{H}_{0}: \rho=0$ vs. $\mathrm{H}_{1}: \rho \neq 0$ is computed using Fisher's z [8] transformation and is given as follows:

$$
t_{\text {Fboot }}=\sqrt{n-3}\left(\mathrm{z}^{*}-\mathrm{z}_{0}\right)
$$

Where critical values of the above statistic is the $z_{\alpha / 2}^{*}$, which is the $(\alpha / 2)^{\text {th }}$ sample quintiles of $t_{\text {Fboot }}$.

\section{Parametric bootstrap version of proposed test statistic}

The test statistic for testing $\mathrm{H}_{0}: \rho=0 v$ s. $\mathrm{H}_{1}: \rho \neq 0$ is computed follows:

$$
t_{S K b o o t}=\frac{r}{\sqrt{\frac{M S E}{\sum_{i=1}^{n}\left(\mathrm{y}_{i-} \overline{\bar{y}}\right)^{2}}}}
$$

Where critical values of the above statistic is the $t_{(\mathrm{n}-2)}^{*}, \frac{\alpha}{2}$, which is the $(\alpha / 2)^{\text {th }}$ sample quintiles of $t_{\text {SKboot }}$.

\section{Bootstrap bias corrected acceleration test statistic}

This method is introduced by Efron and Tibshirani [9]. The test statistic for testing $\mathrm{H}_{0}: \rho=0 v s . \mathrm{H}_{1}: \rho \neq 0$ is the $\mathrm{t}$-statistic defined in eqn. (1) and the critical value is calculated by $t_{B C A}^{c r i}=r^{(\alpha)}$, where $\mathrm{r}^{()}$is the $(100 \alpha) \%$ percentile of the distribution of $r, \alpha=\phi\left[\hat{\mathrm{z}}_{0}+\frac{\hat{z}_{0}+z^{(\alpha)}}{1-\hat{a}\left\{\hat{z}_{0}+z^{(\alpha)}\right\}}\right], \phi($.$) is$ the standard normal cumulative distribution function, bias correction $\hat{z}_{0}=\phi^{-1}\left(\frac{\#\left(\mathrm{r}_{i}^{*}<\mathrm{r}\right)}{B}\right), \phi^{-1}($.$) is the inverse function of cumulative$ distribution function of the $\mathrm{Z}$ distribution, acceleration factor $\hat{a}=\sum_{i=1}^{n}\left(\mathrm{r}-\mathrm{r}_{-i}\right)^{3} / 6\left[\sum_{i=1}^{n}\left(\mathrm{r}-\mathrm{r}_{-i}\right)^{2}\right]^{1.5}, \mathrm{r}$ is the correlation between $\mathrm{x}$ and $y$ and $r_{i}$ is the correlation between $x$ and $y$ of (n-1) observations without the $\mathrm{i}^{\text {th }}$ observation.

\section{Simulation Study}

The main goal of this paper is to evaluate the performance of test statistics for testing population correlation coefficient based on size and power properties, discussed in section 2 . Since a theoretical comparison among the tests is not possible, a simulation study has been conducted in this section.

\section{Simulation design}

MATLAB (2015) programming language was used to run simulations and to make necessary tables. The most common level of significance $\alpha=0.05$ is considered and assumed random sample sizes $\mathrm{n}=10,30,50,80$ and 100 and $\rho_{1}=-0.5,-0.9,0.3,0.8$ and 0.99 . We have considered 2500 replications for our simulation experiments and 1500 bootstrap samples for each selected random samples sizes. Random samples produced from the following population distributions:

(a) Bivariate normal with $\mu_{1}=15, \mu_{2}=20$ and $\sigma_{1}=10, \sigma_{2}=4$

(b) Bivariate $\log$ normal with $\mu_{1}=15, \mu_{2}=20$ and $\sigma_{1}=10, \sigma_{2}=4$

(c) Bivariate gamma with shape parameters 1 and mean parameters 2

(d) Bivariate chi-square with dfs 1 and 3 
Citation: Banik S, Golam Kibria BM (2017) Size and Power Properties of Some Test Statistics for Testing the Population Correlation Coefficient $\rho$. J Biom Biostat 8: 353. doi: 10.4172/2155-6180.1000353

Page 3 of 10

\section{Results Discussion}

Table 1 presents estimated sizes of the selected test statistics for selected values of $\mathrm{n}$ and $\rho$, when random samples are generated from the bivariate normal distribution. For a visual expression, simulation results are presented graphically in Figure 1.

We can see from Figure 1 and Table 1 is that for all sample sizes, all proposed test statistics except GL1 and SK Boot have empirical sizes close to the $5 \%$ nominal level.

We have presented estimated sizes when data are generated from the bivariate lognormal distribution in Table 2 and depicted results for visual inspection in Figure 2. From Table 2 and Figure 2, we observe that for moderate to large sample sizes, tBoot, FBoot, SKBoot and BCABoot have sizes close to the nominal level, while rest of the tests achieve nominal level only when sample sizes are large.

In Tables 3 and 4, we have reported estimated sizes when data generated from the bivariate gamma and bivariate chi-square distribution respectively. We find that all tests have correct sizes expect GL1 and SKboot. GL1 test has small sizes than the nominal level and SKboot has higher sizes than the nominal level (Figures 3 and 4).

In Table 5, we have presented the estimated powers when data are generated from the bivariate normal distribution for various sample sizes and various values of $\rho$. We observed that for small sample size $\mathrm{n}=10$ (Figure 5), Fisher, G0L3, GL4, FBoot and SKBoot have good

\begin{tabular}{|c|c|c|c|c|}
\hline Tests & $\mathbf{n = 1 0}$ & $\mathbf{n = 3 0}$ & $\mathbf{n = 5 0}$ & $\mathbf{n = 8 0}$ \\
\hline $\mathbf{t}$ & 0.0476 & 0.0520 & 0.0480 & 0.0588 \\
\hline Fisher & 0.0488 & 0.0548 & 0.0484 & 0.0604 \\
\hline GL1 & 0.0920 & 0.0608 & 0.0528 & 0.0592 \\
\hline GL2 & 0.0592 & 0.0552 & 0.0512 & 0.0608 \\
\hline GL3 & 0.0440 & 0.0512 & 0.0452 & 0.0560 \\
\hline SK & 0.0248 & 0.0476 & 0.0436 & 0.0564 \\
\hline tBoot & 0.0460 & 0.0360 & 0.0356 & 0.0532 \\
\hline SBoot & 0.0300 & 0.0332 & 0.0336 & 0.040 \\
\hline BCABoot & 0.0424 & 0.0524 & 0.0552 & 0.050 \\
\hline
\end{tabular}

Table 1: Estimated sizes at the $5 \%$ level of significance for bivariate normal data.

$n=10 \square \mathrm{n}=30 \quad \mathrm{n}=50 \quad \mathrm{n}=80 \quad \mathrm{n}=100$

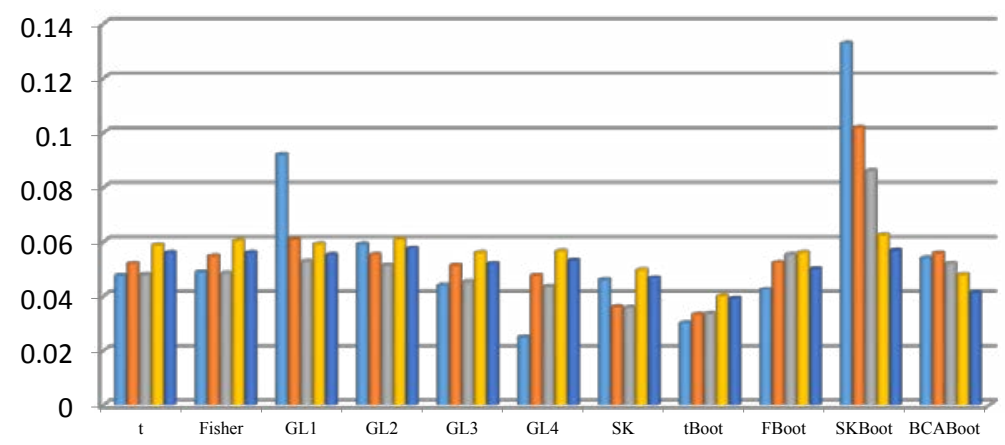

Figure 1: Estimated sizes for various values of $n$ in case of the bivariate normal distribution.

\begin{tabular}{|c|c|c|c|c|}
\hline Tests & $\mathbf{n = 1 0}$ & $\mathbf{n = 3 0}$ & $\mathbf{n = 5 0}$ & $\mathbf{n = 0}$ \\
\hline $\mathbf{t}$ & 0.0896 & 0.0608 & 0.0496 & 0.0436 \\
\hline Fisher & 0.0904 & 0.0608 & 0.0504 & 0.0436 \\
\hline GL1 & 0.1028 & 0.0620 & 0.0508 & 0.0436 \\
\hline GL2 & 0.0960 & 0.0608 & 0.0508 & 0.0436 \\
\hline GL3 & 0.0876 & 0.0604 & 0.0488 & 0.0436 \\
\hline GL4 & 0.0792 & 0.0600 & 0.04312 & 0.0436 \\
\hline tBoot & 0.0896 & 0.0608 & 0.0496 & 0.0312 \\
\hline SBoot & 0.0464 & 0.0312 & 0.0252 & 0.0312 \\
\hline BCABoot & 0.0228 & 0.0356 & 0.0260 \\
\hline
\end{tabular}

Table 2: Estimated sizes at the $5 \%$ level of significance for bivariate lognormal data. 
Citation: Banik S, Golam Kibria BM (2017) Size and Power Properties of Some Test Statistics for Testing the Population Correlation Coefficient $\rho$. J Biom Biostat 8: 353. doi: 10.4172/2155-6180.1000353

$n=10 \quad n=30 \quad \square=50 \quad n=80 \quad \square n=100$

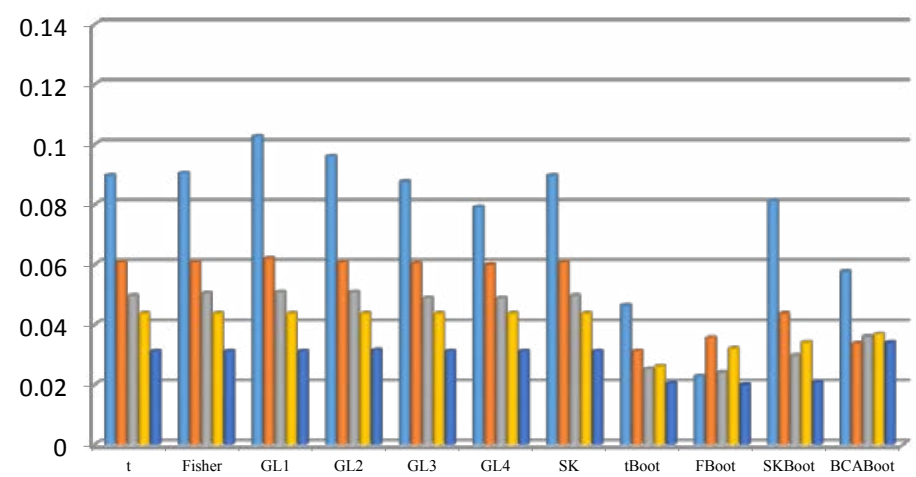

Figure 2: Estimated sizes for various values of $n$ in case of the bivariate lognormal distribution.

\begin{tabular}{|c|c|c|c|c|}
\hline Tests & $\mathbf{n = 1 0}$ & $\mathbf{n = 3 0}$ & $\mathbf{n = 5 0}$ & $\mathbf{n = 0}$ \\
\hline $\mathbf{t}$ & 0.0520 & 0.0500 & 0.0536 & 0.0468 \\
\hline Fisher & 0.0524 & 0.0504 & 0.0540 & 0.0472 \\
\hline GL1 & 0.0924 & 0.0588 & 0.0564 & 0.0472 \\
\hline GL2 & 0.0652 & 0.0532 & 0.0560 & 0.0484 \\
\hline GL3 & 0.0500 & 0.0484 & 0.0492 & 0.0440 \\
\hline SK & 0.0288 & 0.0432 & 0.0480 & 0.0452 \\
\hline tBoot & 0.0520 & 0.0500 & 0.0536 & 0.0468 \\
\hline SBoot & 0.0152 & 0.0276 & 0.0184 & 0.0252 \\
\hline BCABoot & 0.0348 & 0.0368 & 0.0508 & 0.0436 \\
\hline
\end{tabular}

Table 3: Estimated sizes at the $5 \%$ level of significance for the bivariate gamma data.

\begin{tabular}{|c|c|c|c|c|c|}
\hline Tests & $n=10$ & $n=30$ & $n=50$ & $n=80$ & $n=100$ \\
\hline $\mathbf{t}$ & 0.0456 & 0.0452 & 0.0560 & 0.0544 & 0.0468 \\
\hline Fisher & 0.0472 & 0.0456 & 0.0568 & 0.0548 & 0.0468 \\
\hline GL1 & 0.0912 & 0.0504 & 0.0596 & 0.0548 & 0.0468 \\
\hline GL2 & 0.0608 & 0.0480 & 0.0592 & 0.0568 & 0.0476 \\
\hline GL3 & 0.0420 & 0.0428 & 0.0520 & 0.0500 & 0.0448 \\
\hline GL4 & 0.0220 & 0.0388 & 0.0516 & 0.0516 & 0.0460 \\
\hline SK & 0.0456 & 0.0452 & 0.0560 & 0.0544 & 0.0468 \\
\hline tBoot & 0.0064 & 0.0008 & 0.0228 & 0.0312 & 0.0224 \\
\hline FBoot & 0.0348 & 0.0432 & 0.0400 & 0.0408 & 0.0424 \\
\hline SKBoot & 0.2108 & 0.1828 & 0.2000 & 0.1744 & 0.1700 \\
\hline BCABoot & 0.0364 & 0.0344 & 0.0500 & 0.0488 & 0.0392 BCABoot \\
\hline
\end{tabular}

Table 4: Estimated sizes at the $5 \%$ level of significance for the bivariate chi-square data.

powers as compare to other test statistics. For sample sizes 50 or above, (Figure 6 for $\mathrm{n}=50$ ) we found that all test statistics have good powers except for $\rho=0.3$. We noted that for weak positive correlation, SKboot has highest power as compare to rest of the test statistics.

In Figure 7, we have presented estimated powers when data are generated from the bivariate lognormal distribution for $n=10$. We observed that for strong negative correlation, $t$, SK, tBoot and BCABoot tests statistics have very poor power compare to other tests statistics. For positive correlation, we found that all tests have good powers but tBoot and BCABoot. We observed that these two tests have very low powers as compare to other test statistics.

Figure 8 presents estimated powers when data are generated from the bivariate lognormal distribution for $n=80$. We observed that all tests have good powers except bootstrap versions of t, Fisher, SK and BCA for $\rho=0.3$.

we have tabulated estimated power of various test statistics when data are generated from bivariate gamma and bivariate chi-square distributions (see Figure $9(n=10)$ and Figure $10(n=30)$ for better understanding). It is observed that power properties of the selected tests are similar when data generated from bivariate normal distribution or lognormal.

In Figures 11 and 12, we have plotted estimated powers for various values of $n$ for $\rho=-0.5$ and $\rho=0.8$ to check effects of $n$ on the selected tests. It is observed from these graphs that as $\mathrm{n}$ increases powers are 
Citation: Banik S, Golam Kibria BM (2017) Size and Power Properties of Some Test Statistics for Testing the Population Correlation Coefficient $\rho$. J Biom Biostat 8: 353. doi: 10.4172/2155-6180.1000353

Page 5 of 10

$\square \mathrm{n}=10 \quad \mathrm{n}=30 \quad \square \mathrm{n}=50 \quad \square \mathrm{n}=80 \quad \square \mathrm{n}=100$

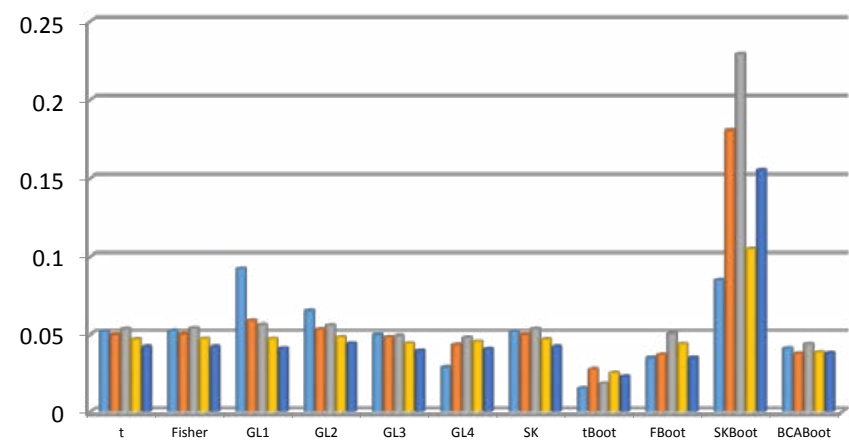

Figure 3: Estimated sizes for various values of $\mathrm{n}$ in case of the bivariate gamma distribution.

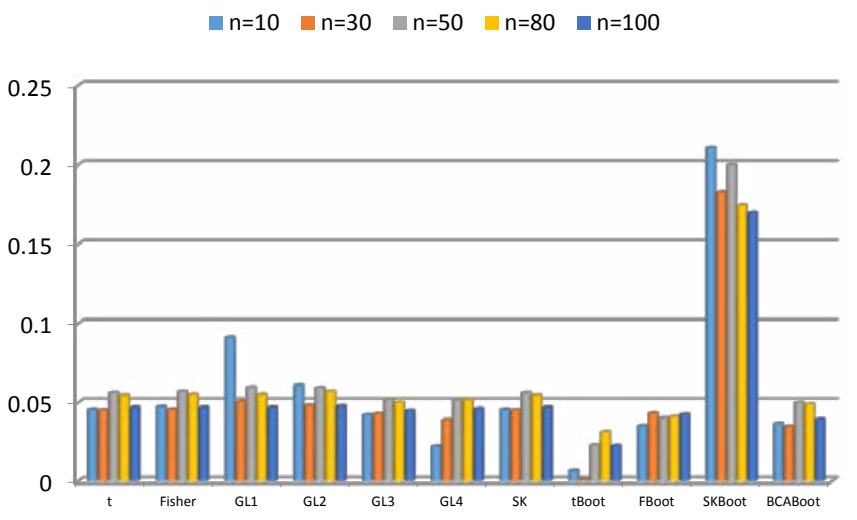

Figure 4: Estimated sizes for various values of $n$ in case of the bivariate chi square distribution.

\begin{tabular}{|c|c|c|c|c|c|c|c|c|c|c|c|}
\hline & $\mathbf{t}$ & Fisher & GL1 & GL2 & GL3 & GL4 & SK & tBoot & FBoot & SKBoot & BCABoot \\
\hline \multirow{2}{*}{ pho $=-0.5$} & & & & & & $n=10$ & & & & & \\
\hline & 0.2360 & 0.3300 & 0.3680 & 0.2860 & 0.3140 & 0.2200 & 0.2360 & 0.1440 & 0.2900 & 0.5800 & 0.2760 \\
\hline pho $=-0.9$ & 0.6000 & 0.9740 & 0.7780 & 0.6820 & 0.9740 & 0.9640 & 0.6000 & 0.4740 & 0.9840 & 0.9700 & 0.7400 \\
\hline pho $=0.3$ & 0.0940 & 0.1040 & 0.1680 & 0.1080 & 0.1020 & 0.0660 & 0.0940 & 0.0420 & 0.0940 & 0.3280 & 0.1080 \\
\hline pho $=0.8$ & 0.4680 & 0.8120 & 0.6260 & 0.5300 & 0.8000 & 0.7220 & 0.4680 & 0.3500 & 0.8460 & 0.8760 & 0.5600 \\
\hline pho $=0.99$ & 0.6620 & 1.0000 & 0.8520 & 0.7620 & 1.0000 & 1.0000 & 0.6620 & 0.4860 & 1.0000 & 0.9420 & 0.6260 \\
\hline \multirow{2}{*}{ pho $=-0.5$} & & & & & & $n=30$ & & & & & \\
\hline & 0.7720 & 0.8420 & 0.7940 & 0.7760 & 0.8320 & 0.8260 & 0.7720 & 0.6660 & 0.8260 & 0.8800 & 0.7320 \\
\hline pho $=-0.9$ & 0.9080 & 0.9080 & 0.9620 & 0.9200 & 0.9080 & 0.9580 & 0.9080 & 0.7560 & 0.9560 & 0.9580 & 0.9080 \\
\hline pho $=0.3$ & 0.2480 & 0.2800 & 0.2720 & 0.2520 & 0.2640 & 0.2480 & 0.2480 & 0.1740 & 0.3180 & 0.5380 & 0.2140 \\
\hline pho $=0.8$ & 0.8440 & 0.9300 & 0.8600 & 0.8500 & 0.9180 & 0.9120 & 0.8440 & 0.6820 & 0.8940 & 0.9300 & 0.8400 \\
\hline pho $=0.99$ & 0.9012 & 0.9745 & 0.9340 & 0.9310 & 0.9235 & 0.9205 & 0.9012 & 0.7930 & 0.9012 & 0.9745 & 0.8923 \\
\hline \multirow{2}{*}{ pho $=-0.5$} & & & & & & $n=50$ & & & & & \\
\hline & 0.9500 & 0.9740 & 0.9520 & 0.9520 & 0.9660 & 0.9660 & 0.9500 & 0.9240 & 0.9440 & 0.9740 & 0.9340 \\
\hline pho $=-0.9$ & 0.9920 & 0.9980 & 0.9920 & 0.9920 & 0.9980 & 0.9980 & 0.9920 & 0.9800 & 0.9960 & 0.9980 & 0.9920 \\
\hline pho $=0.3$ & 0.4420 & 0.4780 & 0.4500 & 0.4460 & 0.4600 & 0.4560 & 0.4420 & 0.3720 & 0.5220 & 0.6960 & 0.4260 \\
\hline pho $=0.8$ & 0.9660 & 0.9900 & 0.9740 & 0.9720 & 0.9900 & 0.9900 & 0.9660 & 0.9420 & 0.9920 & 0.9980 & 0.9540 \\
\hline pho $=0.99$ & 1.0000 & 1.0000 & 1.0000 & 1.0000 & 1.0000 & 1.0000 & 1.0000 & 1.0000 & 1.0000 & 1.0000 & 1.0000 \\
\hline \multirow{2}{*}{ pho $=-0.5$} & & & & & & $n=80$ & & & & & \\
\hline & 0.9940 & 0.9980 & 0.9960 & 0.9960 & 0.9980 & 0.9980 & 0.9940 & 0.9860 & 0.9980 & 0.9980 & 0.9940 \\
\hline pho $=-0.9$ & 0.9960 & 1.0000 & 0.9960 & 0.9960 & 1.0000 & 1.0000 & 0.9960 & 0.9940 & 1.0000 & 1.0000 & 0.9940 \\
\hline pho $=0.3$ & 0.5480 & 0.5860 & 0.5540 & 0.5560 & 0.5740 & 0.5740 & 0.5480 & 0.4420 & 0.5920 & 0.7780 & 0.4780 \\
\hline pho $=0.8$ & 0.9900 & 0.9980 & 0.9900 & 0.9920 & 0.9960 & 0.9960 & 0.9960 & 0.9900 & 0.9780 & 1.0000 & 0.9840 \\
\hline pho $=0.99$ & 1.0000 & 1.0000 & 1.0000 & 1.0000 & 1.0000 & 1.0000 & 1.0000 & 1.0000 & 1.0000 & 1.0000 & 1.0000 \\
\hline \multirow{2}{*}{ pho $=-0.5$} & & & & & & $n=100$ & & & & & \\
\hline & 1.0000 & 1.0000 & 1.0000 & 1.0000 & 1.0000 & 1.0000 & 1.0000 & 1.0000 & 1.0000 & 1.0000 & 1.0000 \\
\hline pho $=-0.9$ & 1.0000 & 1.0000 & 1.0000 & 1.0000 & 1.0000 & 1.0000 & 1.0000 & 1.0000 & 1.0000 & 1.0000 & 1.0000 \\
\hline pho $=0.3$ & 0.7200 & 0.7620 & 0.7200 & 0.7260 & 0.7440 & 0.7540 & 0.7200 & 0.5820 & 0.7020 & 0.8480 & 0.6860 \\
\hline pho $=0.8$ & 1.0000 & 1.0000 & 1.0000 & 1.0000 & 1.0000 & 1.0000 & 1.0000 & 1.0000 & 1.0000 & 1.0000 & 1.0000 \\
\hline pho $=0.99$ & 1.0000 & 1.0000 & 1.0000 & 1.0000 & 1.0000 & 1.0000 & 1.0000 & 1.0000 & 1.0000 & 1.0000 & 1.0000 \\
\hline
\end{tabular}

Table 5: Powers at the $5 \%$ level of significance for bivariate normal data. 
Citation: Banik S, Golam Kibria BM (2017) Size and Power Properties of Some Test Statistics for Testing the Population Correlation Coefficient $\rho$. J Biom Biostat 8: 353. doi: 10.4172/2155-6180.1000353

$\square$ pho=-0.5 pho=-0.9 $\square$ pho=0.3 pho=0.8 $\square$ pho $=0.99$

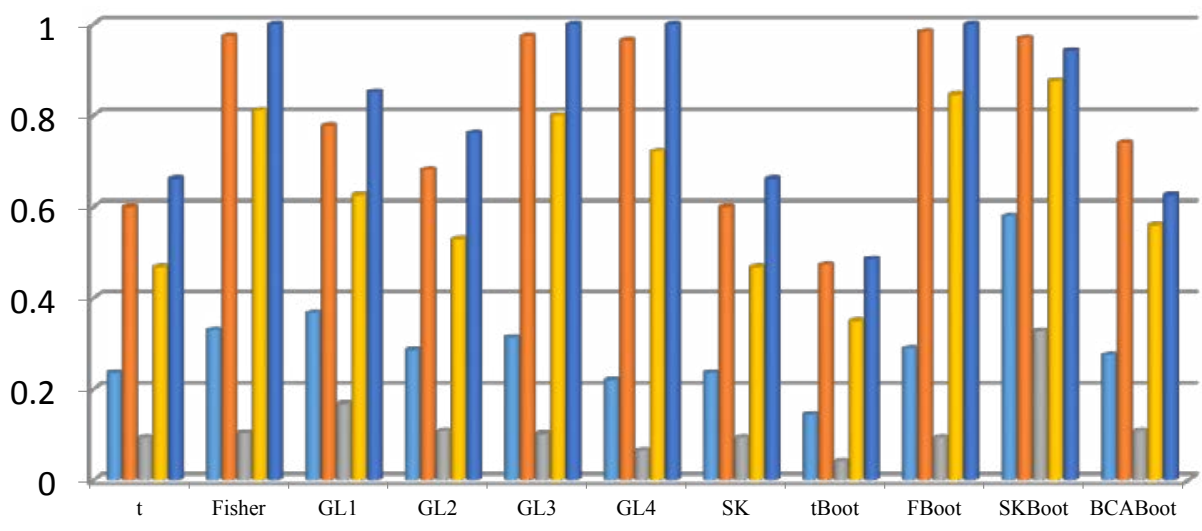

Figure 5: Estimated powers for $n=10$ and various values of $\rho$ for bivariate normal distribution

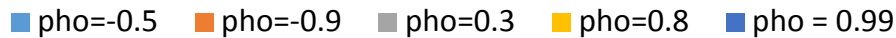

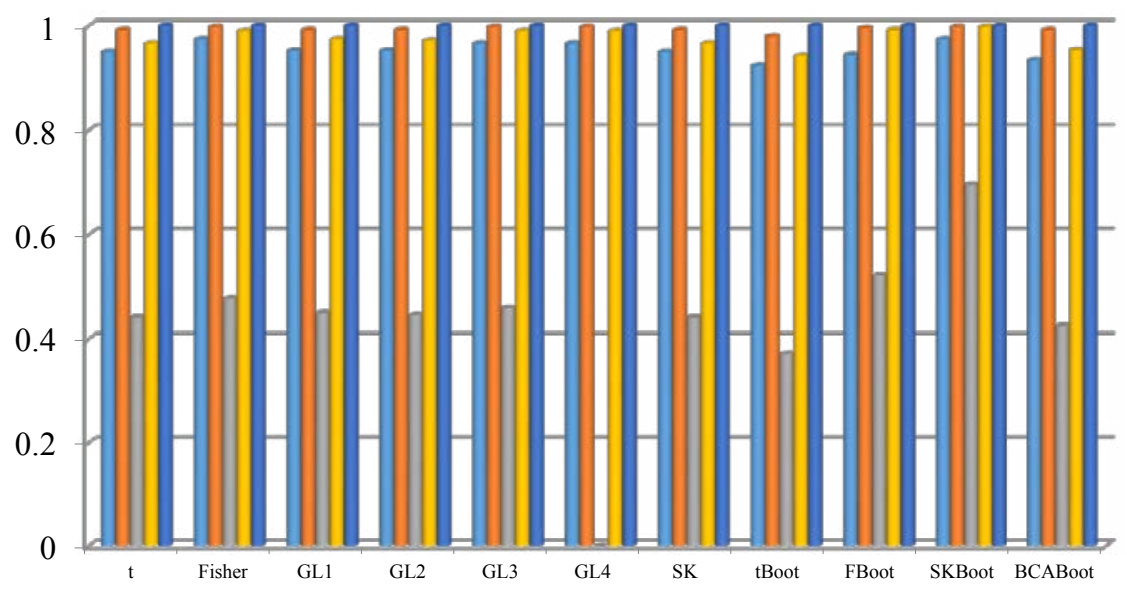

Figure 6: Estimated powers for $n=50$ and various values of $\rho$ in case of bivariate normal distribution.

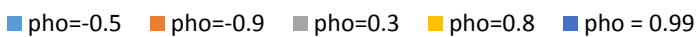

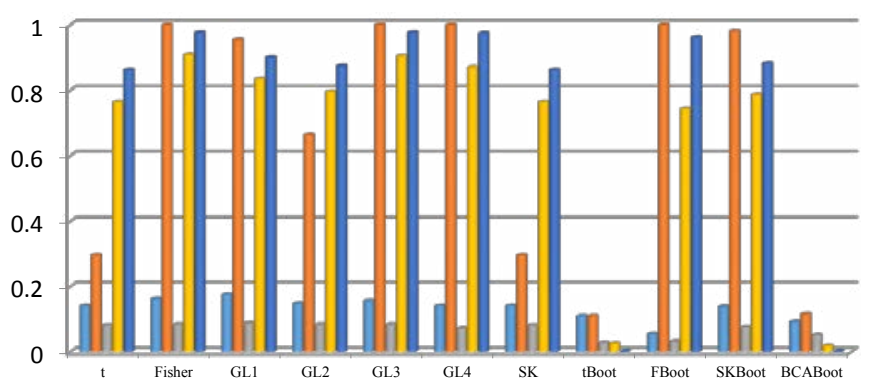

Figure 7: Estimated powers for $n=10$ and various values of $\rho$ in case of bivariate lognormal distribution.
" pho $=-0.5 \square$ pho $=-0.9 \square \mathrm{pho}=0.3 \square \mathrm{pho}=0.8 \square \mathrm{pho}=0.99$

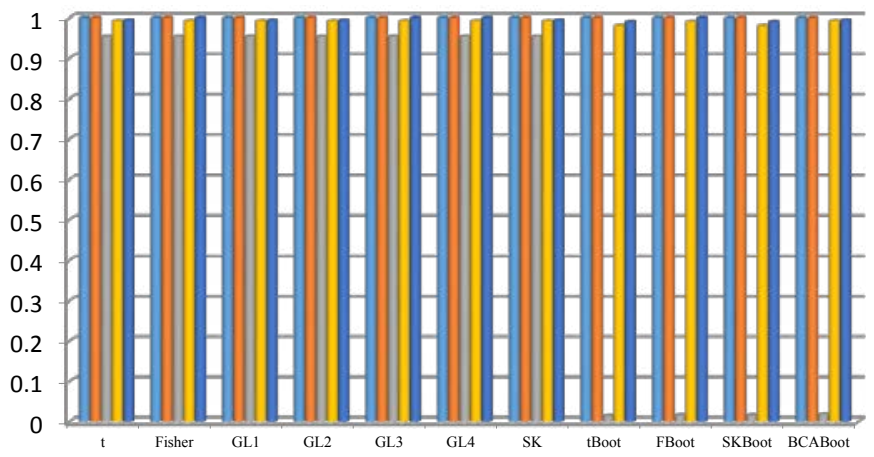

Figure 8: Estimated powers for $n=80$ and various values of $\rho$ in case of bivariate lognormal distribution. 
Citation: Banik S, Golam Kibria BM (2017) Size and Power Properties of Some Test Statistics for Testing the Population Correlation Coefficient $\rho$. J Biom Biostat 8: 353. doi: 10.4172/2155-6180.1000353

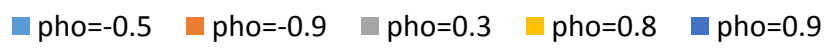

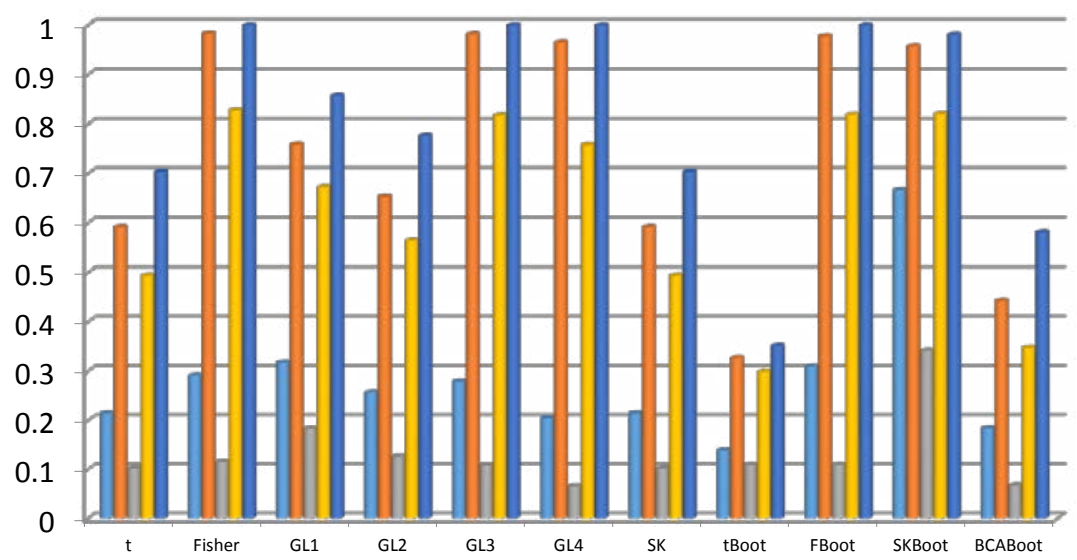

Figure 9: Estimated powers for $n=10$ and various values of $\rho$ for bivariate gamma distribution

$\square$ pho $=-0.5 \square$ pho $=-0.9 \square$ pho $=0.3 \square$ pho $=0.8 \square$ pho $=0.9$

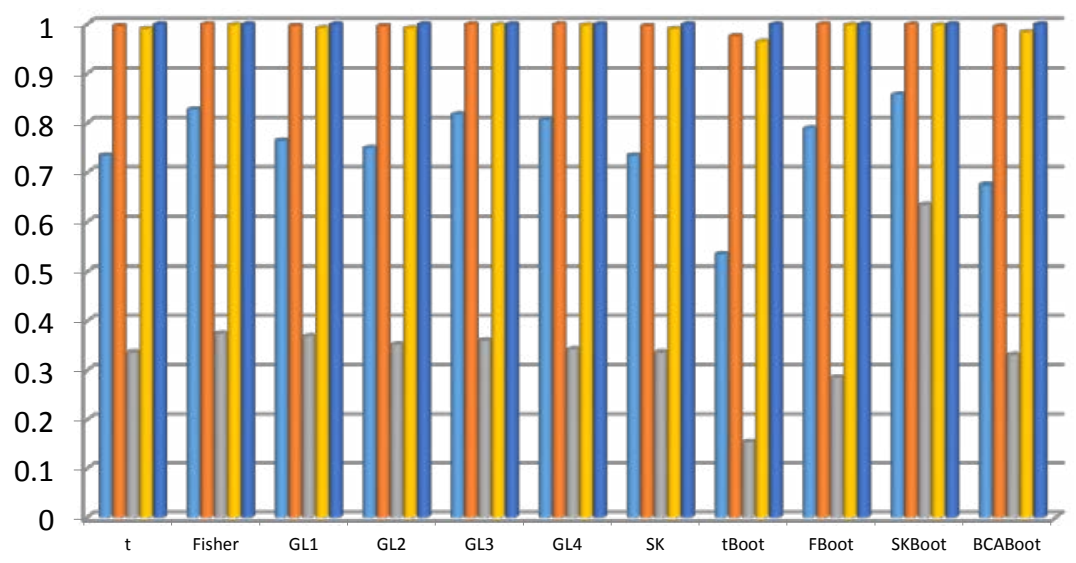

Figure 10: Estimated powers for $n=30$ and various values of $\rho$ in case of bivariate chi-square distribution. $n=10 \square n=30 \square n=50 \square n=80 \quad n=100$

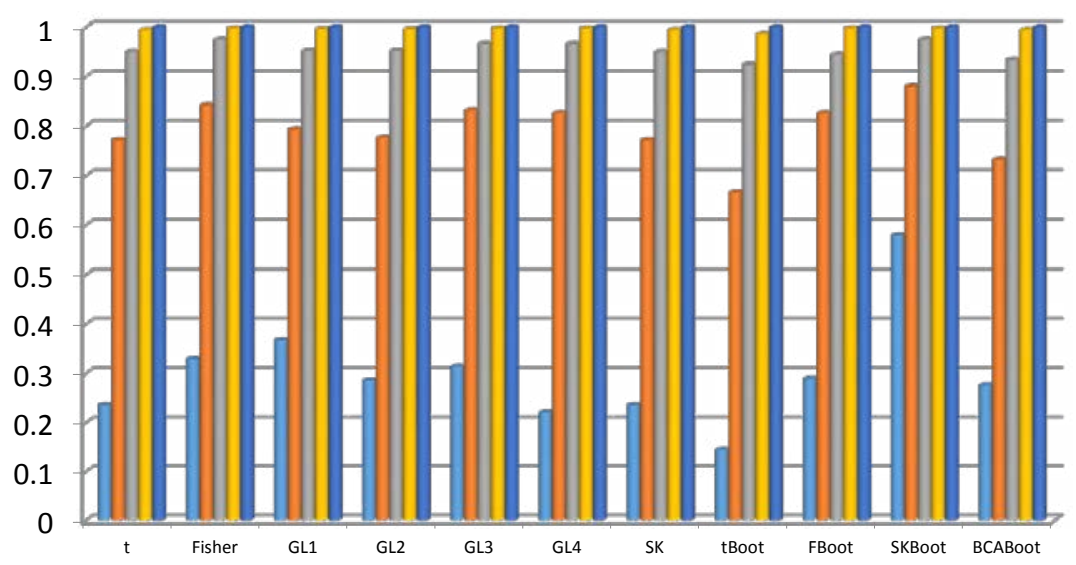

Figure 11: Estimated powers for various values of $n$ and $\rho=-0.5$ in case of bivariate normal distribution. 
Citation: Banik S, Golam Kibria BM (2017) Size and Power Properties of Some Test Statistics for Testing the Population Correlation Coefficient $\rho$. J Biom Biostat 8: 353. doi: 10.4172/2155-6180.1000353

$n=10 \square n=30 \square n=50 \square n=80 \quad n=100$

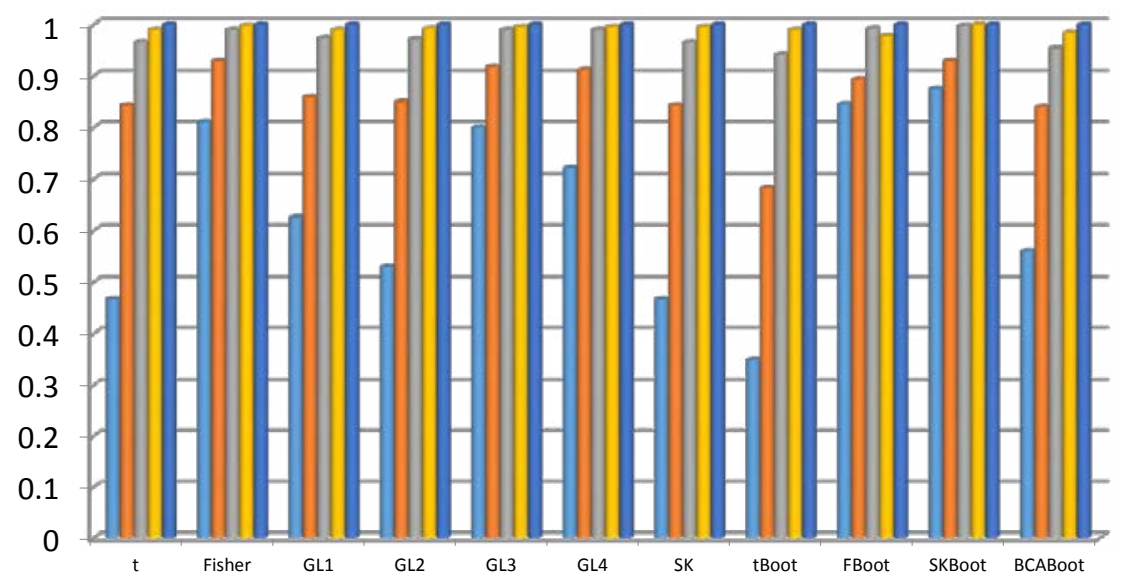

Figure 12: Estimated powers for various values of $n$ and $\rho=0.8$ in case of bivariate normal distribution.

$n=10 \square n=30 \square n=50 \quad n=80 \quad n=100$

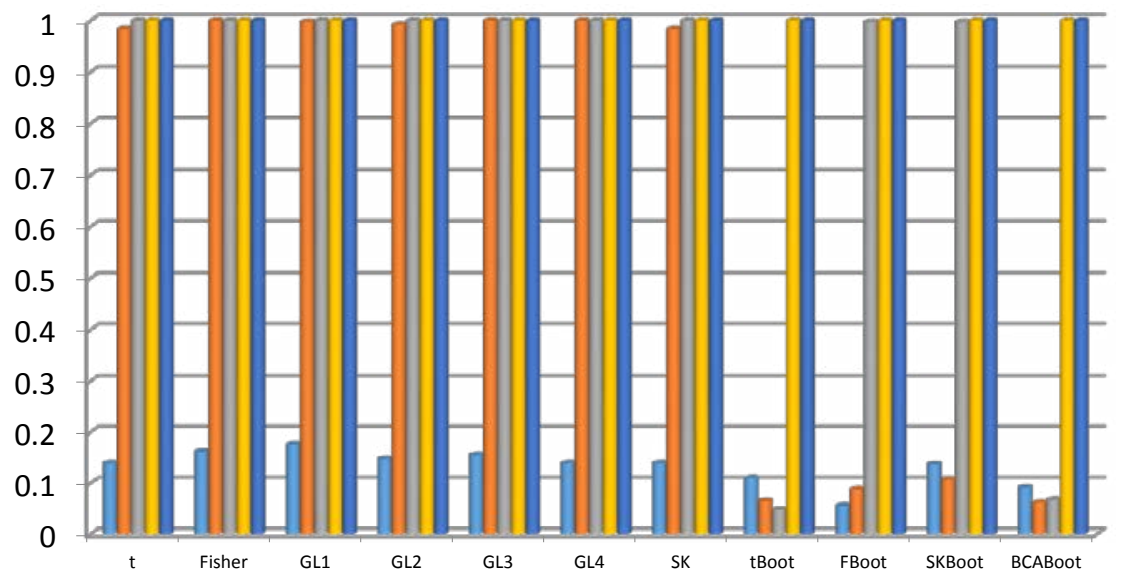

Figure 13: Estimated powers for various values of $n$ and $\rho=-0.5$ and bivariate lognormal distribution.

$n=10 \square n=30 \square n=50 \quad n=80 \quad n=100$

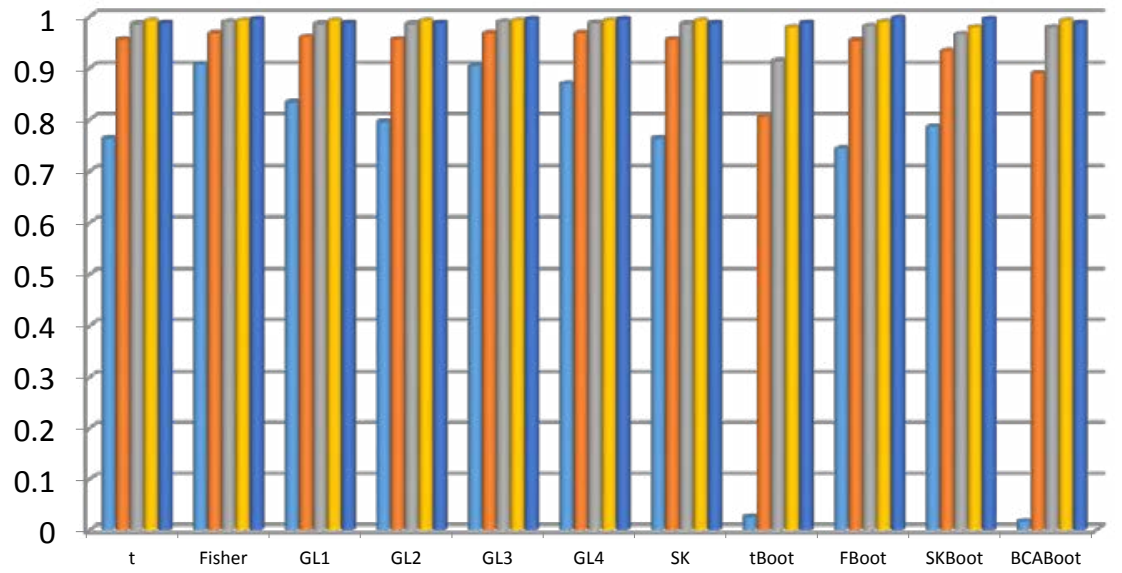

Figure 14: Estimated powers for various values of $n$ and $\rho=0.8$ and bivariate lognormal distribution. 
Citation: Banik S, Golam Kibria BM (2017) Size and Power Properties of Some Test Statistics for Testing the Population Correlation Coefficient $\rho$. J Biom Biostat 8: 353. doi: 10.4172/2155-6180.1000353

Page 9 of 10

also increases for all selected tests. We noted that for small sample sizes, Fisher, GL2, Fboot and SKboot tests are more powerful than the other considered tests. It is also noted that our proposed bootstrap version SKboot is more powerful than the other considered tests.

In Figures 13 and 14, we have plotted estimated powers for selected values of $\mathrm{n}$ and two selected values of $\rho$. Here also we observed same patterns like Figures 11 and 12 as sample size increases, estimated powers also increases. As compare to the Figure 11, we noted very low powers $\mathrm{n}=10$ when data generated from the bivariate lognormal distribution. We noted that tboot and BCAboot tests have very low power compared to the other tests.
Figures 15-18 present estimated powers for various values of $\mathrm{n}$ and two selected values of $\rho$ when data are generated from the bivariate gamma distribution and bivariate chi-square distribution respectively. Similar interpretation can be drawn from these figures, as we observed when data are generated from the bivariate normal distribution.

\section{Conclusion}

In this paper, we study the performance of several methods for testing the population correlation coefficient by means of a simulation study. Data were generated randomly from several bivariate distributions, namely, bivariate normal, bivariate lognormal, bivariate

$n=10 \square \mathrm{n}=30 \quad \mathrm{n}=50 \quad \mathrm{n}=80 \quad \square \mathrm{n}=100$

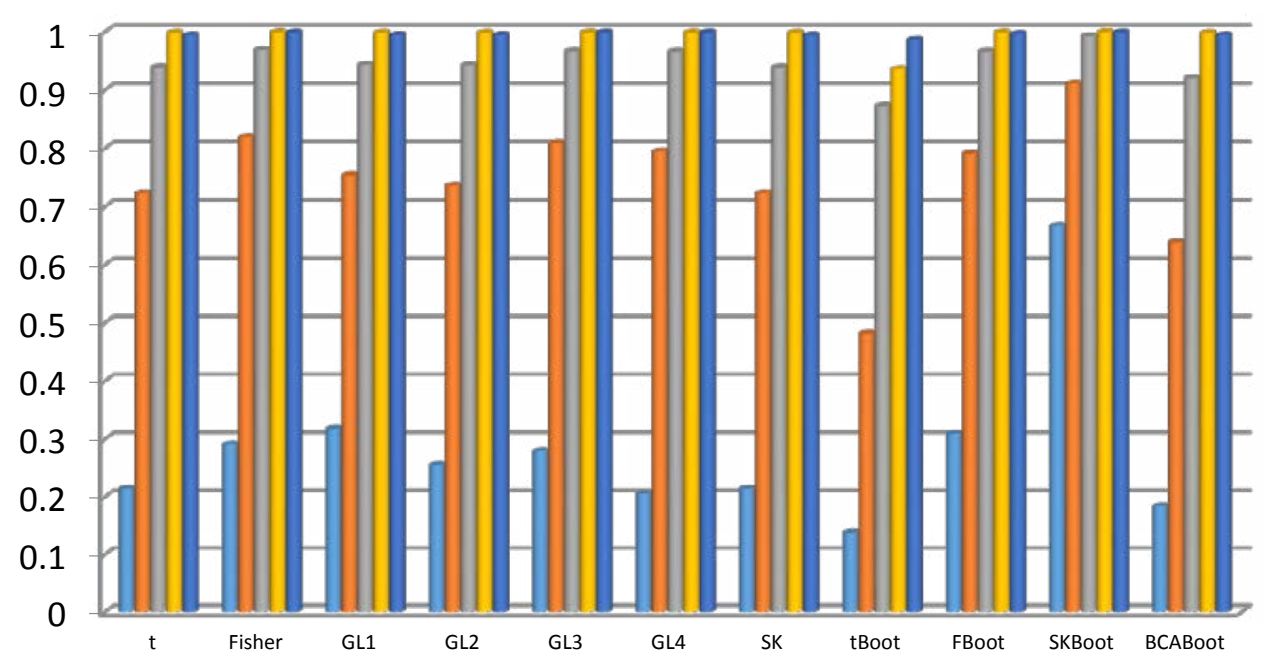

Figure 15: Estimated powers for various values of $n$ and $\rho=-0.5$ in case of bivariate gamma distribution.

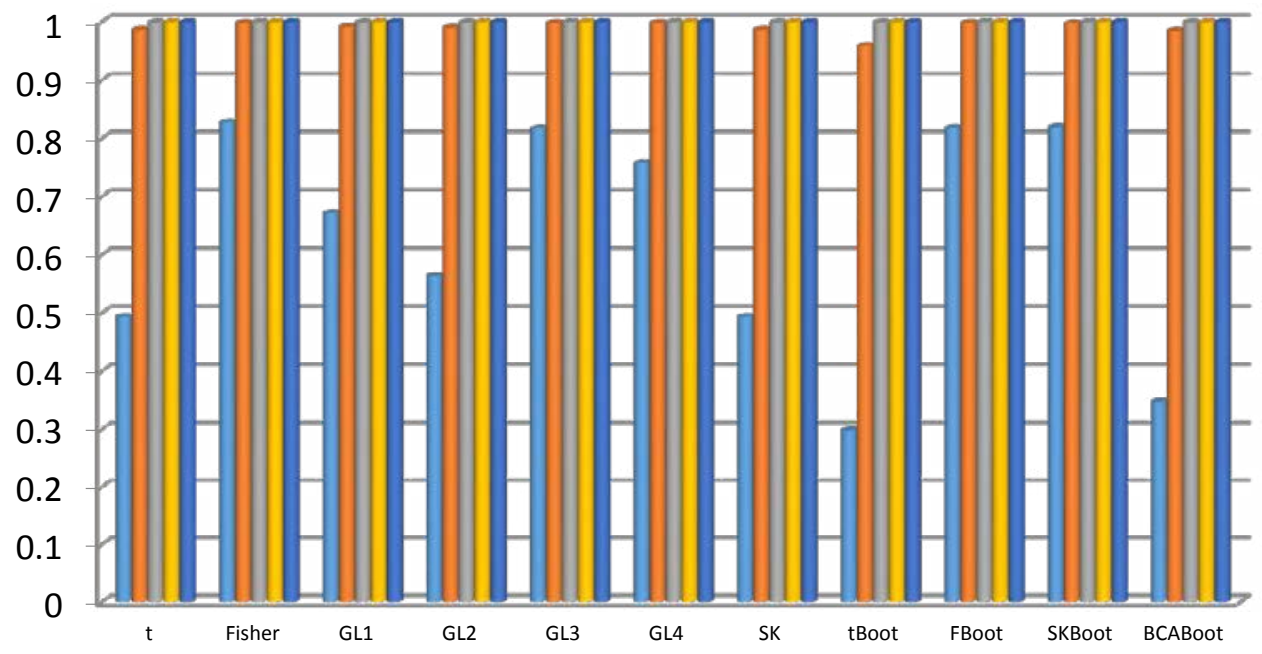

Figure 16: Estimated powers for various values of $n$ and $\rho=0.8$ in case of bivariate gamma distribution. 
Citation: Banik S, Golam Kibria BM (2017) Size and Power Properties of Some Test Statistics for Testing the Population Correlation Coefficient $\rho$. J Biom Biostat 8: 353. doi: 10.4172/2155-6180.1000353

$$
n=10-n=30-n=50 \quad n=80 \quad n=100
$$

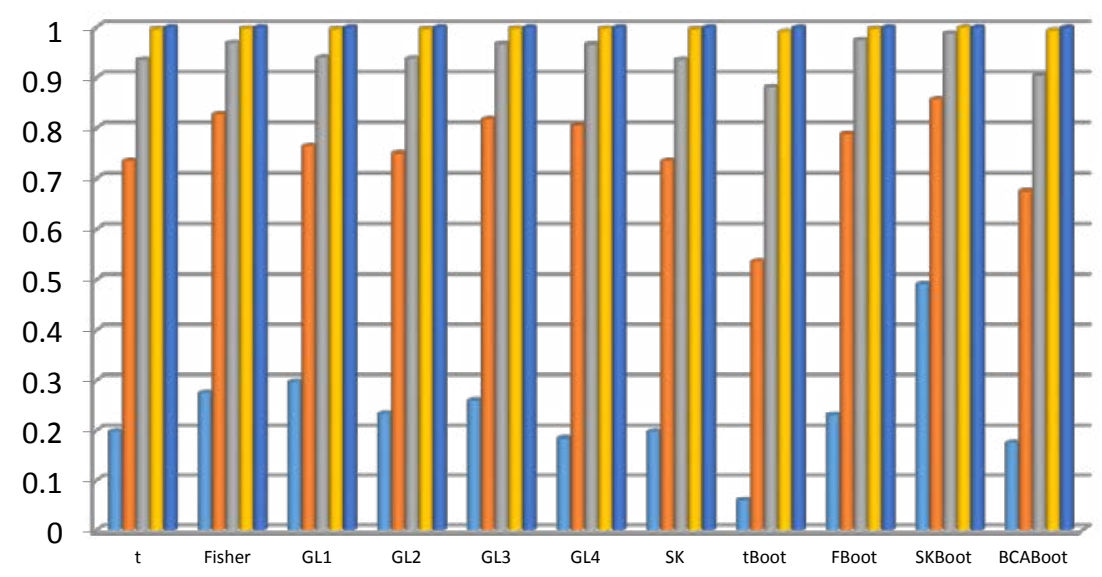

Figure 17: Estimated powers for various values of $n$ and $\rho=-0.5$ in case of bivariate chi-square distribution.

$n=10 \square n=30 \quad n=50 \quad n=80 \quad n=100$

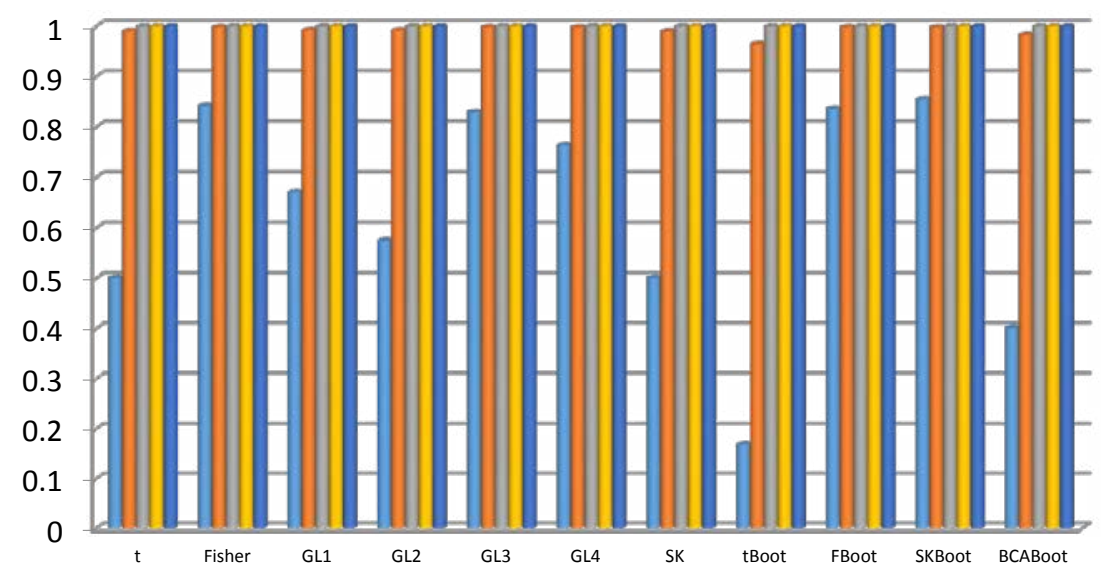

Figure 18: Estimated powers for various values of $\mathrm{n}$ and $\rho=0.8$ in case of bivariate chi-square distribution.

gamma and bivariate chi-square with a range of sample sizes. Overall, we found that test statistics, t, Fisher, GL2, GL3, GL4, SK and FBoot have sizes close to the $5 \%$ nominal level. Fisher, GL3, GL4, FBoot and SKBoot have good powers as compare to other test statistics. It appears from the simulation study is that the test statistics, Fisher, GL3, GL4, FBoot and SKBoot can be recommended for practitioners because these test statistics have good sizes and powers compare to the rest of selected test statistics.

\section{References}

1. Pearson K (1896) Mathematical Contributions to the Theory of Evolution III Regression Heredity and Panmixia. Philosophical Transactions of the Royal Society of London 187: 253-318.

2. Galton $F(1886)$ Regression towards mediocrity in hereditary stature. Journal of the Anthropological Institute of Great Britain and Ireland 15: 246-263.

3. Banik S, Kibria BMG (2016) Confidence Intervals for the population correlation coefficients $\rho$. International Journal of Statistics in Medical Research 5: 99-111.

4. Student (1908) The Probable Error of a Correlation Coefficient. Biometrica 6: 302-310.

5. Fisher RA (1915) Frequency Distribution of the Values of the Correlation
Coefficient in Samples from an Indefinitely Large Population. Biometrica 10: 507-521.

6. Gorsuch RL, Lehmann CS (2010) Correlation Coefficient Mean Bias and Confidence Interval Distortions. Journal of Methods and Measurement in the Social Science 1: 52-65.

7. Efron B (1979) Bootstrap Methods another Look at the Jack knife. Annals of Statistics 7: 1- 26.

8. Fisher RA. (1921) On the Probable Error of a Coefficient of Correlation Deduced from a Small Sample. Metron1: 3-32.

9. Efron B, Tibshirani RJ (1993) An Introduction to the Bootstrap Chapman \& Hall New York. 